\title{
BMJ Open Mean arterial pressure for predicting preeclampsia in Asian women: a longitudinal cohort study
}

\author{
Jing Zhu, ${ }^{1,2}$ Jun Zhang (D) , ${ }^{1,3}$ Nurul Syaza Razali, ${ }^{3}$ Bernard Chern, ${ }^{3}$ Kok Hian Tan $^{3}$
}

To cite: Zhu J, Zhang J, Syaza Razali N, et al.

Mean arterial pressure for predicting preeclampsia in Asian women: a longitudinal cohort study. BMJ Open 2021;11:e046161. doi:10.1136/ bmjopen-2020-046161

- Prepublication history and additional supplemental material for this paper are available online. To view these files, please visit the journal online. (http://dx.doi.org/10.1136/ bmjopen-2020-046161)

Received 22 October 2020 Accepted 22 July 2021

Check for updates

(c) Author(s) (or their employer(s)) 2021. Re-use permitted under CC BY-NC. No commercial re-use. See rights and permissions. Published by BMJ.

${ }^{1}$ Ministry of Education-Shanghai Key Laboratory of Children's Environmental Health, Xinhua Hospital, Shanghai Jiao Tong University School of Medicine, Shanghai, China

${ }^{2}$ Department of Obstetrics and Gynecology, Xinhua Hospital, Shanghai Jiao Tong University School of Medicine, Shanghai, China

${ }^{3}$ Division of Obstetrics and Gynaecology, KK Women's and Children's Hospital, Singapore

Correspondence to

Dr Jun Zhang:

junjimzhang@sina.com

\section{ABSTRACT}

Objective Previous studies suggested mean arterial pressure (MAP) had moderate predictive values in the first and second trimesters for the prediction of preeclampsia. However, the performance of MAP in Asian women is still unclear. The objective of this study was to examine the predictive values of MAP in Asian population throughout gestation, and to compare the performance of MAP, angiogenic factors and uterine artery Doppler in the prediction of preeclampsia.

Design A prospective cohort study.

Setting KK Women's and Children's Hospital, Singapore. Participants A total of 926 women with singleton pregnancy less than 14 weeks of gestation were included in the prospective Neonatal and Obstetrics Risks Assessment cohort between September 2010 and October 2014. Maternal blood pressure levels, uterine artery pulsatility index (UtA-PI), serum soluble fms-like tyrosine kinase 1 (sFlt-1), placental growth factor (PIGF) and sFlt-1/ PIGF ratio were measured at 11-14, 18-22, 28-32 and 34 weeks onward, respectively.

Primary and secondary outcomes Preeclampsia was the main pregnancy outcome.

Results A total of 20 women developed preeclampsia, who had significantly lower levels of PIGF, higher levels of sFIt-1/PIGF ratio and MAP throughout pregnancy than women without preeclampsia. Compared with angiogenic factors and UtA-PI, MAP had significantly higher area under the receiver operating characteristic curves (AUCs) for predicting preeclampsia and term preeclampsia throughout gestation. For predicting preeclampsia, MAP had AUCs of 0.86 (95\% Cl 0.78 to 0.95$), 0.87$ (95\% Cl 0.80 to 0.95$)$ and $0.91(95 \% \mathrm{Cl} 0.85$ to 0.98$)$ at $11-14$, 18-22 and 28-32 weeks, respectively. For predicting term preeclampsia, MAP yielded AUCs of 0.87 (95\% Cl 0.75 to $0.99), 0.87$ (95\% Cl 0.76 to 0.98$)$ and 0.90 (95\% Cl 0.80 to 0.99 ) at 11-14, 18-22 and 28-32 weeks, respectively. For predicting preterm preeclampsia, the performance of MAP and PIGF was similar.

Conclusion MAP is a good predictor for preeclampsia, especially term preeclampsia, in Asian women.

\section{INTRODUCTION}

Preeclampsia, along with other hypertensive disorders of pregnancy, is one of the leading causes of maternal death in both developed and developing countries every year, complicating $2 \%-8 \%$ of pregnancies. ${ }^{12}$

\section{Strengths and limitations of this study}

This study was based on a well-performed prospective cohort in Asian population with standard protocols on measurements of maternal blood pressure, circulating angiogenic factors and uterine artery Doppler throughout gestation.

- The performance of mean arterial pressure, angiogenic factors and uterine artery Doppler for predicting preeclampsia was assessed and compared by the area under the receiver operating characteristic curve.

- The effect of white coat hypertension and relatively low incidence of preeclampsia in this cohort might impact the performance of biomarkers in predicting preeclampsia.

Early identification of women at risk can facilitate prenatal surveillance and management. Currently, risk factors from maternal demographic characteristics and medical history are used to identify women at high risk of developing preeclampsia. ${ }^{3}{ }^{4}$ However, maternal risk factors alone only predicted one third of cases. ${ }^{5}$

The published screening approaches showed that the first-trimester combined test using Bayes theorem to combine a priori risk from maternal characteristics and medical history with biomarkers, could increase the detection rate of preeclampsia, especially the preterm preeclampsia. ${ }^{67}$ However, the performance of the combined test for prediction of preeclampsia was poor in Asian population. ${ }^{89}$ Besides, resources of placental growth factor (PIGF) measurement and uterine artery Doppler might be limited in some developing areas. Hence, evaluation of maternal risk factors and blood pressure measurement could be a pragmatic approach to identify women at risks. ${ }^{10}$

Previous studies suggested a moderate predictive value of mean arterial pressure (MAP) in the first and second trimesters. $^{11}{ }^{12}$ But various blood pressure measurement protocols were carried out 
in these studies and most of the study population were Caucasian. Lower MAP and PIGF levels in Asian women, alongside with lower incidence of preeclampsia, may influence the efficiency of predictive models. ${ }^{13}{ }^{14}$ It is still unclear regarding the predictive value of MAP in the first, second and third trimesters for predicting preeclampsia in Asian women, and how the performance of MAP is in comparison with angiogenic factors and uterine artery Doppler. Thus, we used data from a prospective cohort study to evaluate the performance of MAP in predicting preeclampsia in Asian population, and to compare the performance of predictors during pregnancy.

\section{MATERIALS AND METHODS \\ Study population}

The Neonatal and Obstetrics Risks Assessment (NORA) study was a prospective cohort conducted at the KK Women's and Children's Hospital in Singapore between September 2010 and October 2014. ${ }^{15}$ Singleton pregnancies less than 14 weeks of gestation were recruited during the study period. The exclusion criteria were multiple gestations, chronic medical conditions such as renal disease or systemic lupus erythematosus and pregnancies complicated by aneuploidy or fetal anomaly. Gestational age was confirmed from the fetal crown-rump length. Detailed interviews, biophysical measurements, ultrasound scans and blood sample collections were performed at recruitment (11-14 weeks), 18-22 weeks, 28-32 weeks and 34 weeks onward, respectively. Information on pregnancy complications, labour and delivery and neonatal outcomes was collected through medical chart review. A written informed consent was obtained from all participating women.

A total of 1013 women were enrolled initially and 934 of them completed all 4 antenatal visits. We excluded 8 participants without pregnancy outcomes, leaving 926 participants for the final analysis (online supplemental figure 1).

\section{Definitions}

Preeclampsia was defined according to the guidelines of International Society for the Study of Hypertension in Pregnancy ${ }^{16}$ : systolic blood pressure (SBP) $\geq 140 \mathrm{~mm} \mathrm{Hg}$ and/or diastolic blood pressure (DBP) $\geq 90 \mathrm{~mm} \mathrm{Hg}$ on at least two occasions 4 hours apart after 20 weeks of gestation in a previously normotensive women, and proteinuria: urinary albumin $\geq 300 \mathrm{mg} / 24$ hours urine collection or $\geq 1+$ dipstick. For women with chronic hypertension, superimposed preeclampsia was defined as a significant increase in blood pressure compared with baseline (30 $\mathrm{mm} \mathrm{Hg}$ systolic, $15 \mathrm{~mm} \mathrm{Hg}$ diastolic) in association with new-onset proteinuria. If proteinuria was present at baseline, superimposed preeclampsia was diagnosed if there was doubling of urinary protein excretion after 20 weeks of gestation in association with a significant increase in blood pressure. Superimposed preeclampsia was also diagnosed if blood pressure was elevated and there were elevated liver enzymes (two times baseline) and a low platelet count $\left(<100 \times 10^{9} / \mathrm{L}\right)$. Preeclampsia or superimposed preeclampsia was subdivided according to gestational age at delivery into term ( $\geq 37$ weeks) and preterm term ( $<37$ weeks). Gestational hypertension was defined as newly onset hypertension after 20 weeks of gestation without proteinuria.

Based on the US Preventive Services Task Force, ${ }^{3}$ we classified women as high-risk, moderate-risk and lowrisk groups by maternal demographic characteristics and medical history. High-risk group included women with history of preeclampsia, chronic hypertension, diabetes mellitus or autoimmune disease; moderaterisk group included women with more than one of the following factors: obesity (body mass index $\geq 30 \mathrm{~kg} / \mathrm{m}^{2}$ ), maternal age over 35 years, nulliparity, family history of preeclampsia and previous adverse outcome, including preterm birth and low birth weight; the rest of the participants were considered as the low-risk group. As several studies showed that in women with preeclampsia, especially in those requiring early delivery, uterine artery pulsatility index (UtA-PI) was increased from early pregnancy ${ }^{17-19}$ Women with UtA-PI (11-14 weeks) over the 95th percentile for gestation was also included in the high-risk group.

\section{Laboratory assay}

Maternal venous blood was collected by venipuncture using $10 \mathrm{~mL}$ non-heparinised tubes at each antenatal visit. Serum was isolated by centrifugation at 2000 revolutions/ min for $15 \mathrm{~min}$ and stored at $-80^{\circ} \mathrm{C}$ for subsequent analysis. Samples were measured for soluble fms-like tyrosine kinase 1 (sFlt-1) and PlGF by means of the fully automated Elecsys assays on an electrochemiluminescence immunoassay platform (cobas e411 analyzers, Roche Diagnostics). The detection limit was approximately $6 \mathrm{pg} / \mathrm{mL}$ for sFlt- 1 and $<2 \mathrm{pg} / \mathrm{mL}$ for PlGF.

\section{Uterine artery Doppler measurement}

Doppler ultrasound examinations were carried out transabdominally. At 11-14 weeks of gestation, a sagittal section of the uterus was obtained and colour flow mapping was used to identify each uterine artery along the side of the cervix and uterus at the level of the internal os. ${ }^{20}$ At $18-22$ weeks, 28-32 weeks and 34 weeks onward, colour Doppler was used to identify each uterine artery at the apparent crossover with the external iliac arteries. ${ }^{21}$ Pulsed-wave Doppler was used with the sampling gate set at $2 \mathrm{~mm}$ to cover the whole vessel. When three similar waveforms were obtained consecutively, the pulsatility index (PI) and the resistance index (RI) were measured and mean PI and mean RI of the left and right arteries were calculated, respectively.

\section{Blood pressure measurement}

Blood pressure was taken by validated automated devices (Omron HEM 705 LP, Omron Healthcare) which were calibrated periodically. Women were in a sitting position 
and their arms were supported. A correct cuff size was used and the middle of cuff was positioned on woman's upper arm at the level of the right atrium. ${ }^{22}$ Either a small $(<22 \mathrm{~cm})$, normal $(22-32 \mathrm{~cm})$ or large $(33-42 \mathrm{~cm})$ adult cuff was used depending on the mid-arm circumference. After a rest for $5 \mathrm{~min}$, blood pressure was measured by trained nurses and three recordings were made at $1 \mathrm{~min}$ intervals. We calculated SBP and DBP as the average of the three measurements. MAP was calculated from SBP and DBP measures using the following formula: $\mathrm{MAP}=\mathrm{DBP}+1 / 3 \times(\mathrm{SBP}-\mathrm{DBP})$.

\section{Statistical analysis}

Normality of continuous variables was assessed by the Kolmogorov-Smirnov test. Numeric data were expressed as mean (SD) or as median (IQR) for normally and nonnormally distributed data, respectively. Maternal characteristics and pregnancy outcomes were compared between women with preeclampsia and without preeclampsia using Wilcoxon tests for continuous variables and $\chi^{2}$ analysis for categorical variables. The distributions of sFlt- 1 , PlGF and sFlt-1/PIGF ratio were transformed logarithmically to approximate Gaussian distribution. Covariance analysis was used to compare differences in logarithmtransformed angiogenic factors values, blood pressure levels and uterine artery Doppler values between women with preeclampsia and without preeclampsia adjusted for covariants. Geometric means and $95 \%$ CIs were calculated by taking the exponent of the logarithm transformed mean. If variables did not fit the sphericity tests, repeated measure analysis of variance was performed to examine the differences of variables at four time points between women with preeclampsia and without preeclampsia. The performance of sFlt-1, PIGF, sFlt-1/PIGF ratio, MAP and UtA-PI for the prediction of preeclampsia was assessed by the area under the receiver operating characteristic curve (AUC). Areas were compared using the method of DeLong et al. ${ }^{23}$ Cut-off values were determined by the maximising the sum of sensitivity and specificity. We used SAS V.9.4 for all statistical analyses.

\section{Patient and public involvement}

Patients and the public were not directly involved in the design, conduct or reporting in our study.

\section{RESULTS}

Table 1 compares the characteristics between women with preeclampsia and without preeclampsia. The association of maternal characteristics and preeclampsia is presented in online supplemental table 1. Among 926 participants, 20 women developed preeclampsia, with 8 cases of preterm preeclampsia and 12 cases of term preeclampsia. Women with preeclampsia had a significantly higher proportion of obesity, chronic hypertension and previously history of preeclampsia. Also, higher proportion of preterm birth and lower birth weight was found in women with preeclampsia. Table 2 compares
Table 1 Maternal characteristics and pregnancy outcomes between preeclampsia and non-preeclampsia in the Neonatal and Obstetrics Risks Assessment cohort

\begin{tabular}{|c|c|c|c|}
\hline Variables & $\begin{array}{l}\text { Preeclampsia } \\
(\mathrm{n}=20)\end{array}$ & $\begin{array}{l}\text { Non- } \\
\text { preeclampsia } \\
(n=906)\end{array}$ & $P$ value \\
\hline $\begin{array}{l}\text { Maternal age (year), } \\
\text { median (IQR) }\end{array}$ & $\begin{array}{l}33.0(28.0- \\
37.0)\end{array}$ & $30.0(27.0-34.0)$ & 0.078 \\
\hline Race, n (\%) & & & 0.743 \\
\hline Chinese & $10(50.0)$ & $460(50.8)$ & \\
\hline Indian & $2(10.0)$ & $98(10.8)$ & \\
\hline Malay & $7(35.0)$ & $243(26.8)$ & \\
\hline Others & $1(5.0)$ & $105(11.6)$ & \\
\hline Parity, n (\%) & & & 0.265 \\
\hline 0 & $11(55.0)$ & $490(54.1)$ & \\
\hline 1 & $4(20.0)$ & $291(32.1)$ & \\
\hline$\geq 2$ & $5(25.0)$ & $125(13.8)$ & \\
\hline \multicolumn{3}{|c|}{ Maternal education levels, $n(\%)$} & 0.233 \\
\hline $\begin{array}{l}\text { Less than high } \\
\text { school }\end{array}$ & $8(40.0)$ & $213(23.6)$ & \\
\hline High school & $6(30.0)$ & $361(40.0)$ & \\
\hline College and above & $6(30.0)$ & $329(36.4)$ & \\
\hline Married, n (\%) & $20(100.0)$ & $844(93.2)$ & 0.391 \\
\hline $\begin{array}{l}\text { Smoking during } \\
\text { pregnancy, } \mathrm{n}(\%)\end{array}$ & $1(5.0)$ & $23(2.5)$ & 0.412 \\
\hline \multicolumn{3}{|c|}{ Maternal BMI at $11-14$ weeks of gestation $\left(\mathrm{kg} / \mathrm{m}^{2}\right), \mathrm{n}(\%)$} & 0.041 \\
\hline$<18.5$ & $1(5.0)$ & $63(7.0)$ & \\
\hline $18.5-24.9$ & $7(35.0)$ & $516(57.3)$ & \\
\hline $25.0-29.9$ & $6(30.0)$ & $223(24.8)$ & \\
\hline$\geq 30.0$ & $6(30.0)$ & $99(11.0)$ & \\
\hline $\begin{array}{l}\text { Chronic hypertension, } \\
\text { n (\%) }\end{array}$ & $3(15.0)$ & $7(0.8)$ & 0.001 \\
\hline $\begin{array}{l}\text { Diabetes mellitus, } n \\
(\%)\end{array}$ & $1(5.0)$ & $13(1.4)$ & 0.265 \\
\hline $\begin{array}{l}\text { Previous } \\
\text { preeclampsia, n (\%) }\end{array}$ & $2(10.0)$ & $12(1.3)$ & 0.035 \\
\hline \multicolumn{4}{|l|}{ Pregnancy outcomes } \\
\hline $\begin{array}{l}\text { Gestational age at } \\
\text { delivery, median } \\
\text { (IQR) }\end{array}$ & $\begin{array}{l}37.6(35.0- \\
38.9)\end{array}$ & $39.0(38.1-39.7)$ & $<0.001$ \\
\hline Preterm birth, n (\%) & $8(40.0)$ & $54(6.0)$ & $<0.001$ \\
\hline $\begin{array}{l}\text { Birth weight }(\mathrm{kg}) \text {, } \\
\text { median (IQR) }\end{array}$ & $\begin{array}{l}2.56(2.21- \\
3.03)\end{array}$ & $\begin{array}{l}3.13(2.86- \\
3.40)\end{array}$ & $<0.001$ \\
\hline
\end{tabular}

$\mathrm{BMI}$, body mass index; IQR, interquartile range.

the means and $95 \%$ CIs of biomarkers at four time points during pregnancy between women with preeclampsia and without preeclampsia. Overall, women who developed preeclampsia had significantly lower serum PIGF levels and higher sFlt-1/PlGF ratio and MAP levels throughout pregnancy than those without preeclampsia. However, UtA-PI and serum sFlt-1 levels in women with preeclampsia were increased from the second and third trimesters, respectively. 
Table 2 Covariance analysis of maternal biochemical and biophysical markers at four time points during pregnancy in the Neonatal and Obstetrics Risks Assessment cohort

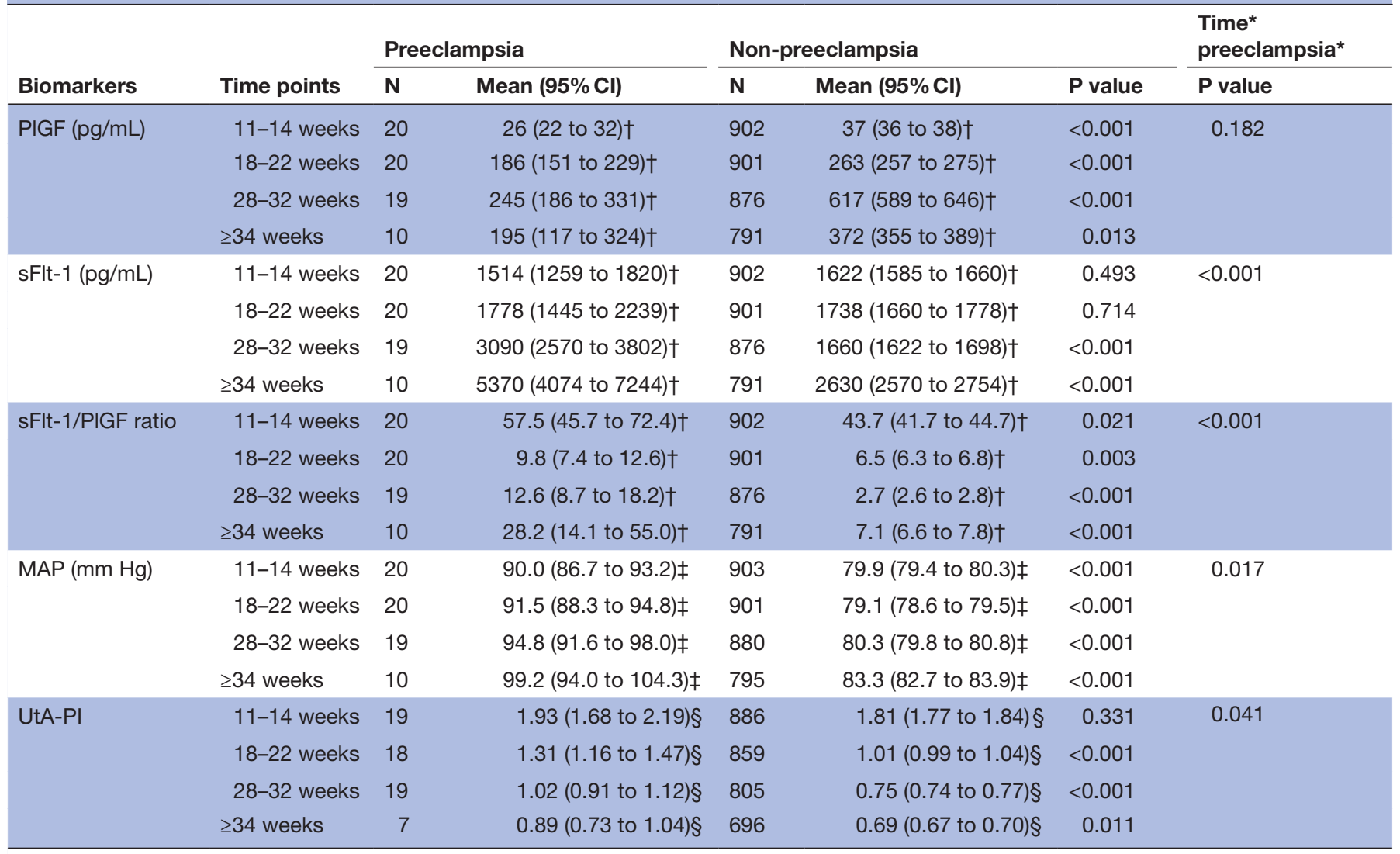

${ }^{*}$ Repeated measure analysis of variance.

†Means $(95 \% \mathrm{Cl})$ are adjusted for maternal age, race, smoking during pregnancy, maternal body mass index at blood test and gestational weeks at blood test from models with logarithm-transformed serum angiogenic factors levels as outcomes; presented as geometric means.

†Means $(95 \% \mathrm{Cl})$ are adjusted for maternal age, race, smoking during pregnancy, maternal body mass index at measurement of blood pressure and uterine artery Doppler, and gestational weeks at measurement of blood pressure and uterine artery Doppler.

$\S$ Means $(95 \% \mathrm{Cl})$ are adjusted for maternal age, race, smoking during pregnancy, maternal body mass index at measurement of blood pressure and uterine artery Doppler, mean arterial pressure at measurement of uterine artery Doppler and gestational weeks at measurement of blood pressure and uterine artery Doppler.

$\mathrm{Cl}$, confidence interval; MAP, mean arterial pressure; PIGF, placental growth factor; sFlt-1, soluble fms-like tyrosine kinase 1; UtA-PI, uterine artery pulsatility index.

Table 3 presents the AUCs of PlGF, sFlt-1, sFlt-1/PlGF ratio, MAP and UtA-PI levels for predicting preeclampsia by weeks of gestation. In 11-14 weeks of gestation, MAP yielded an AUC of 0.86 (95\% CI 0.78 to 0.95 ), which was significantly higher than AUCs of PlGF, sFlt-1, sFlt-1/ PIGF ratio and UtA-PI. Despite that predictive values of angiogenic factors increased from AUC 0.59 in the first trimester to 0.87 in the third trimester, AUC of MAP rose to 0.91 (95\% CI 0.85 to 0.98 ) at 28-32 weeks of gestation, significantly higher than those of angiogenic factors and UtA-PI. Overall, the predictive value of angiogenic factors was low in early pregnancy (AUC 0.59 to 0.67 ) and moderate in middle and late pregnancy (AUC 0.68 to $0.87)$; UtA-PI also had low-to-moderate predictive values during pregnancy (AUC 0.56 to 0.74), while MAP had high predictive values throughout pregnancy (AUC 0.86 to 0.91 ). The performance of prediction models included multiple factors is shown in online supplemental tables 2 and 3. Overall, models that included MAP, PlGF, sFlt-1 and
UtA-PI did not significantly improve the performance of predicting preeclampsia, compared with models included MAP alone.

Table 4 shows the performance of biomarkers in predicting preterm preeclampsia and term preeclampsia during pregnancy. For the prediction of preterm preeclampsia, there was no significant difference of AUCs between MAP and PIGF at 11-14 weeks of gestation, but MAP had a significantly higher AUC than UtA-PI. In the second and third trimesters, the AUCs were not significantly different among predictors. However, for the prediction of term preeclampsia, MAP performed significantly better (AUC $0.87,95 \%$ CI 0.75 to 0.99 ) than other predictors at 11-14 weeks of gestation, and the performance sustained to late pregnancy, with AUC 0.87 (95\% CI 0.76 to 0.98 ) in 18-22 weeks of gestation and AUC 0.90 (95\% CI 0.80 to 0.99 ) in 28-32 weeks of gestation.

We further categorised study population into the high-risk, moderate-risk and low-risk women according 
Table 3 Performance of biomarkers in prediction of preeclampsia during pregnancy in the Neonatal and Obstetrics Risks Assessment cohort

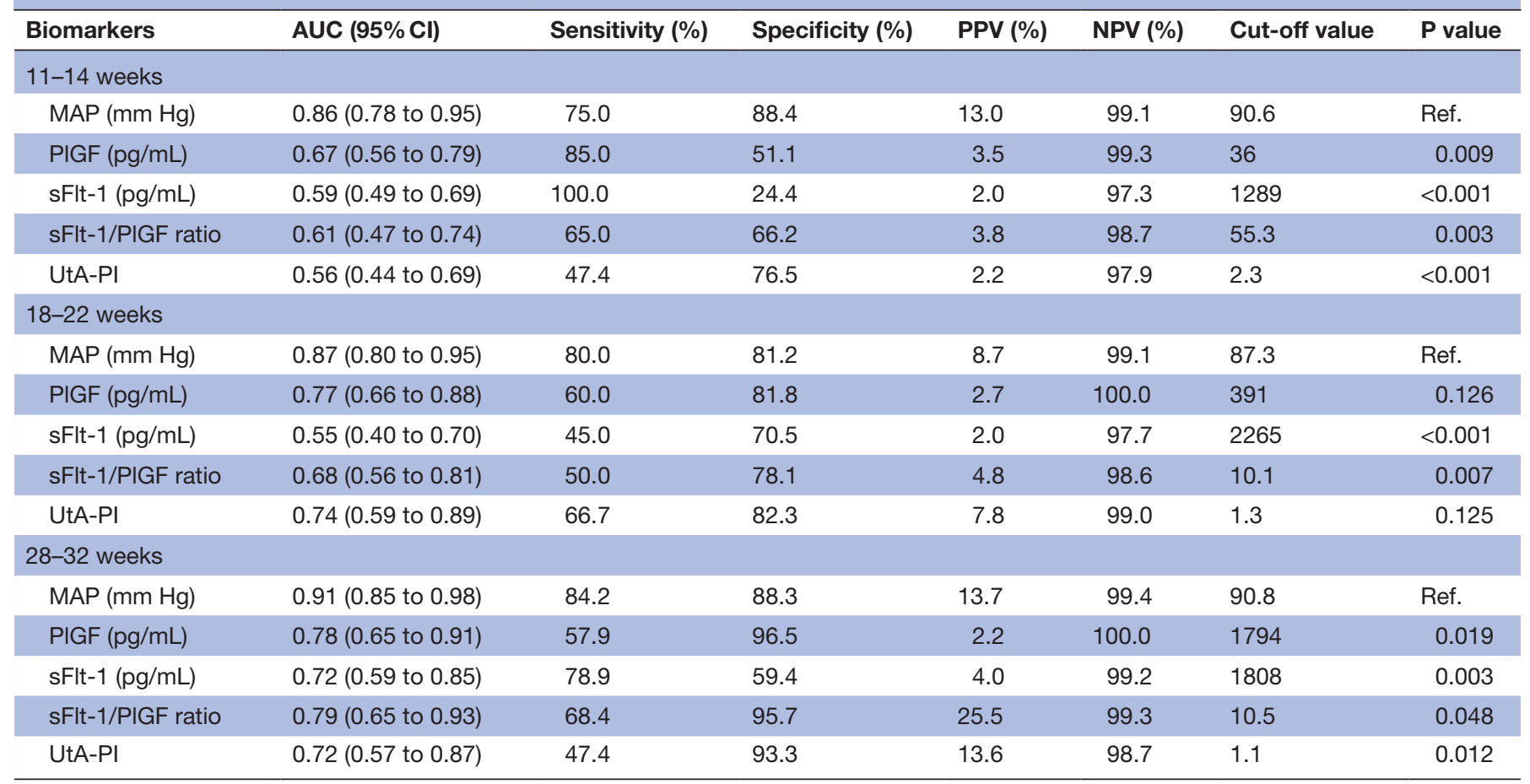

Cut-off values were determined by the maximising the sum of sensitivity and specificity.

AUC, area under the receiver operating characteristic curve; $\mathrm{Cl}$, confidence interval; MAP, mean arterial pressure; NPV, negative predictive value;

PIGF, placental growth factor; PPV, positive predictive value; sFlt-1, soluble fms-like tyrosine kinase 1; UtA-PI, uterine artery pulsatility index.

to the US Preventive Services Task Force and UtA-PI levels in 11-14 weeks of gestation. Based on our definition, the number of women at high, moderate and low risks of preeclampsia was 89,146 and 691, respectively. Characteristics are shown in online supplemental table 4. AUCs of biomarkers in prediction of preeclampsia were performed in high-risk, moderate-risk and lowrisk women, respectively. In high-risk women, MAP and angiogenic factors performed moderate predictive values at 11-14 weeks of gestation, while MAP yielded higher predictive values than other predictors at 18-22 weeks and 28-32 weeks of gestation, with AUCs 0.92 and 0.96, respectively (figure $1 \mathrm{~A}-\mathrm{C}$ ). In moderate-risk women, the performance of MAP in predicting preeclampsia was high throughout pregnancy, with AUCs $0.97,0.95$ and 0.94 at the first, second and third trimester, respectively. Angiogenic factors performed high predictive values just at 28-32 weeks of gestation (figure 1D-F). In low-risk women, MAP performed better than angiogenic factors at 11-14 weeks of gestation (figure 1G-I).

\section{DISCUSSION}

\section{Principal findings of the study}

Our large prospective cohort study in Asian population suggested that compared with angiogenic factors and UtA-PI levels, MAP had significantly higher AUCs at 11-14, 18-22 and 28-32 weeks of gestation for prediction of preeclampsia, especially for the term preeclampsia. For the prediction of preterm preeclampsia, MAP and PlGF were similar. Moreover, in high-risk women, MAP, PlGF and UtA-PI had equivalent predictive values at 11-14 weeks of gestation; in moderate-risk and low-risk women, MAP predicted better than PIGF and UtA-PI in early pregnancy. Our study also confirmed that women who developed preeclampsia, compared with women without preeclampsia, had significantly lower levels of PIGF, higher levels of sFlt-1/PIGF ratio and MAP throughout pregnancy.

\section{Interpretation of the results}

A previous meta-analysis including more than 60 000 women suggested that MAP yielded an AUC of 0.79 in the first trimester and 0.76 in the second trimester. But most of the studies did not report single or multiple measurements of blood pressure. ${ }^{11}$ Poon et al showed that MAP at $11-13^{+6}$ weeks alone had an AUC of 0.734 for prediction of preeclampsia. Considering the interarm blood pressure differences, they measured blood pressure in both arms simultaneously and recorded the last two stable measurements. ${ }^{24}{ }^{25}$ Previous study suggested that the overall screening performance using MAP determined from either the left or right arm, or the average of both arms was not significantly different. But when the average of two or three recordings were used for calculating MAP, the performance of screening tended to improve with an increasing number of recordings. ${ }^{26}$ Although we measured blood pressure in one arm, three readings were recorded at 1-min intervals. Our results 


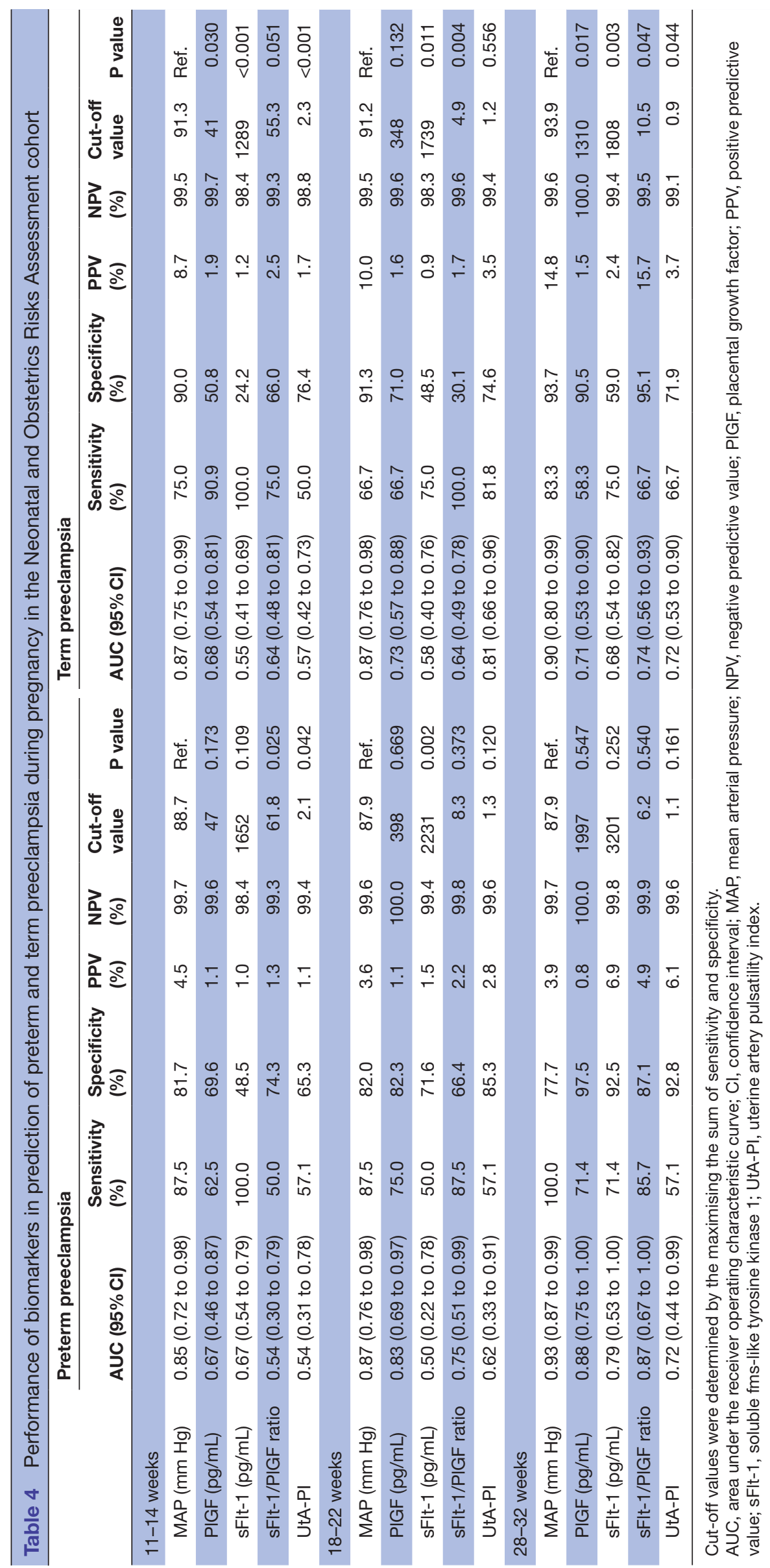




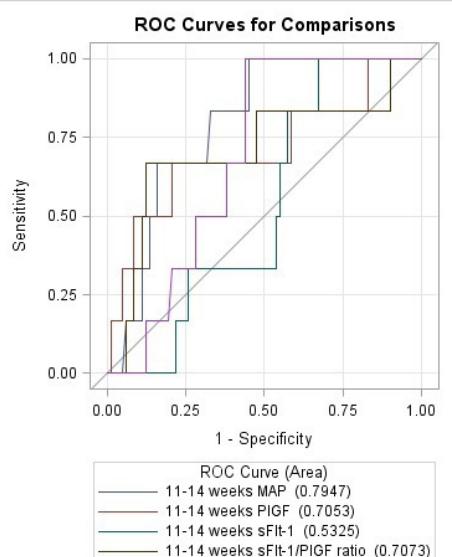

A

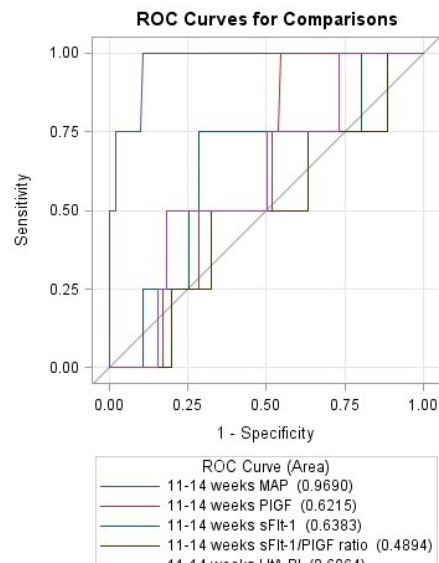

D

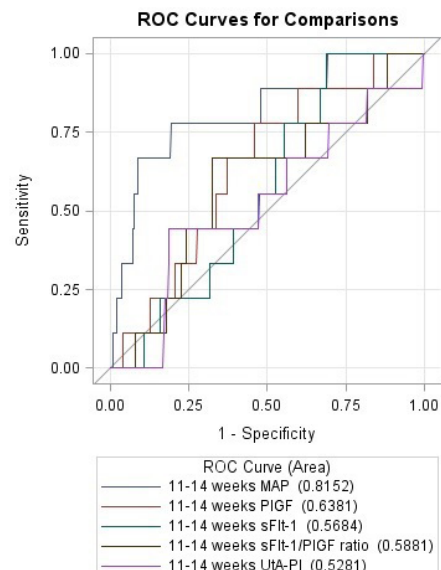

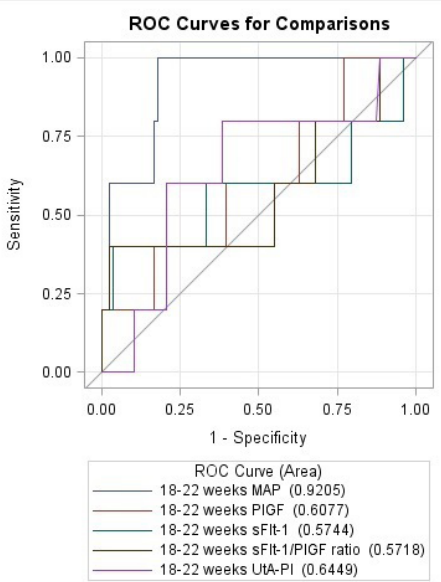

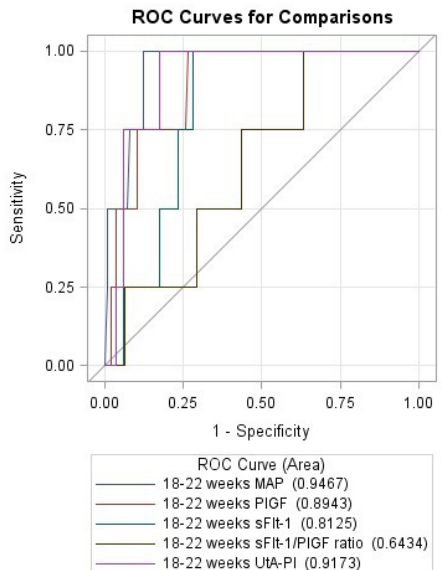

$E$

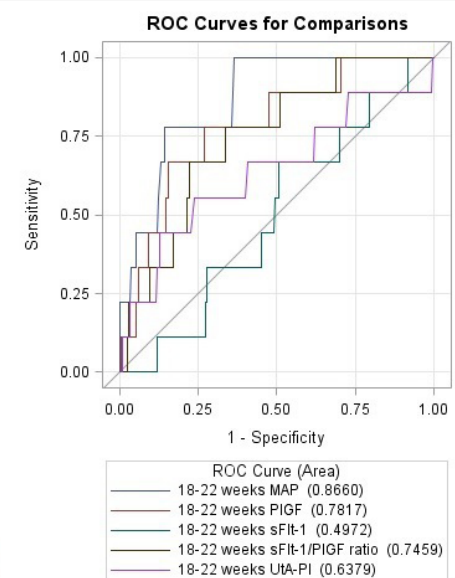

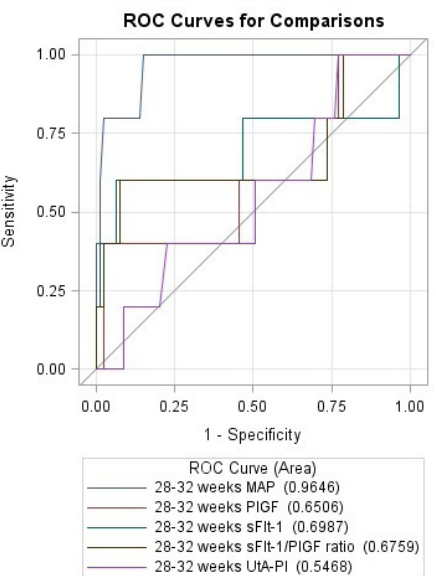

C
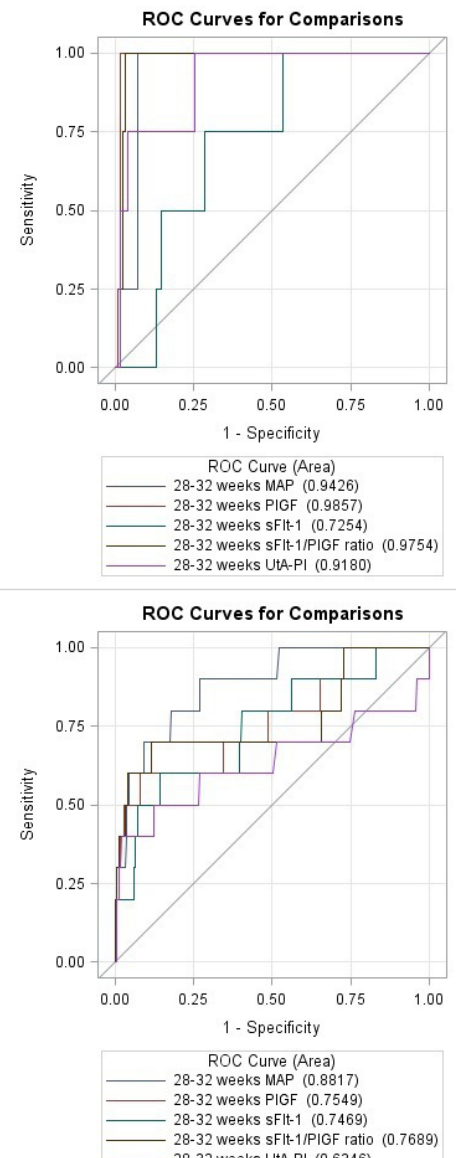

Figure 1 Area under the receiver operating characteristic curves (AUC) of biomarkers for the prediction of preeclampsia. (A) AUC of biomarkers in high-risk women at 11-14 weeks of gestation. (B) AUC of biomarkers in high-risk women at 18-22 weeks of gestation. (C) AUC of biomarkers in high-risk women at 28-32 weeks of gestation. (D) AUC of biomarkers in moderate-risk women at 11-14 weeks of gestation. (E) AUC of biomarkers in moderate-risk women at 18-22 weeks of gestation. (F) AUC of biomarkers in moderate-risk women at 28-32 weeks of gestation. (G) AUC of biomarkers in low-risk women at 11-14 weeks of gestation. (H) AUC of biomarkers in low-risk women at 18-22 weeks of gestation. (I) AUC of biomarkers in low-risk women at 28-32 weeks of gestation. MAP, mean arterial pressure; PIGF, placental growth factor; sFlt-1, soluble fms-like tyrosine kinase 1.

showed that MAP in Asian women had moderate to high performance for the prediction of preeclampsia, with AUCs of 0.86 (95\% CI 0.78 to 0.95 ), 0.87 (95\% CI 0.80 to $0.95)$ and $0.91(95 \%$ CI 0.85 to 0.98$)$ at $11-14,18-22$ and 28-32 weeks, respectively.

Meanwhile, in our Asian population, angiogenic factors showed low to moderate values for the prediction of preeclampsia, with AUCs for PIGF 0.67 (95\% CI 0.56 to 0.79 ), 0.77 (95\% CI 0.66 to 0.88 ), and 0.78 (95\% CI 0.65 to 0.91$)$ at $11-14,18-22$ and $28-32$ weeks, respectively. Our results were similar to those in a longitudinal study which showed AUCs of 0.53 (95\% CI 0.48 to 0.58 ), 0.57 (95\% CI 0.51 to 0.63 ), 0.66 (95\% CI 0.61 to 0.71 ) and $0.72(95 \%$ CI 0.67 to 0.77$)$ at $10,17,24$ and 35 weeks of 
gestation, respectively, for $\mathrm{PlGF}{ }^{27} \mathrm{PlGF} / \mathrm{sFlt}-1$ ratio also showed similar performance as PlGF in the mixed population. ${ }^{27}$ Thus, individual angiogenic factors did not perform well in predicting preeclampsia.

The Fetal Medicine Foundation proposed a combined screening test, including maternal factors, MAP, PIGF and UtA-PI, which was found to predict $75 \%$ of preterm preeclampsia and $47 \%$ of term preeclampsia at a falsepositive rate of $10 \%$ in European population. ${ }^{6}$ However, this algorithm was less predictive in Asian population. ${ }^{89}$ Except for the under-measurement of UtA-PI, the lower incidence of preterm preeclampsia and prevalence of maternal risk factors in Asian women might reduce the effectiveness of the algorithm. ${ }^{9}$ Studies that clarify the causes of the racial differences in preeclampsia are few. Difference in metabolic profiles during 26-28 gestational weeks between White European and South Asian women suggested that White European women had higher levels of lipoproteins, cholesterol, glycerides, phospholipids and monosaturated fatty acids than South Asian women. ${ }^{29}$ A systematic review, examining the association between the endothelial nitric oxide synthase polymorphism and preeclampsia risk stratified by ethnicity, identified a higher risk of preeclampsia in the white or mixed populations with the $894 \mathrm{G}>\mathrm{T}$ polymorphism, but not in those of Asian or African descent. ${ }^{30}$ Another systematic review reported that IL10 -1082 G>A polymorphism significantly increased the risk of preeclampsia in Asian women, but not in White population. ${ }^{31}$ Besides impaired placentation, immunological factors, endothelial function, and inflammation have all been identified as contributors to the pathophysiology of preeclampsia. ${ }^{32}$ Although circulating PIGF and MAP mainly reflect placentation and maternal endothelial function, respectively, racial disparities on pathophysiology might influence the effectiveness of the predictors. As our study showed that while MAP performed better than PIGF for the prediction of preeclampsia and term preeclampsia throughout gestation, for the prediction of preterm preeclampsia, these predictors were not significantly different.

\section{Clinical implications}

Blood pressure measurement has been used as a routine screening and diagnostic tool in antenatal care for decades. Although the American Heart Association statement on blood pressure measurement has been commonly accepted worldwide, which arm to measure and how to record the measurements were not specified in the recommendation. ${ }^{22}$ Our results, along with other studies, suggested that multiple recordings of blood pressure could improve the accuracy for estimating risk of hypertensive disorders. Although we measured blood pressure on one arm, three continuous recordings at 1-min interval could produce an AUC of 0.86 (95\% CI 0.78 to 0.95 ) with $13.0 \%$ positive predictive value at $11-14$ weeks of gestation. This performance in Asian women was better than that in European where blood pressure was measured on both arms with three recordings (AUC
$0.773,95 \%$ CI 0.768 to 0.778$)$. Thus, except for a proper position, appropriately sized cuff and regularly checked devices, multiple recordings should also be emphasised in future clinical practice.

As to the differences between MAP and PlGF in predicting preeclampsia, the predictive values for preterm preeclampsia were not significantly different between MAP and PIGF in the Asian population. In addition, MAP performed better in the prediction of term preeclampsia. Considering the costs and performance of circulating PlGF level for the prediction of preeclampsia, our results support the pragmatic guide for the firsttrimester screening of preeclampsia by the International Federation of Gynecology and Obstetrics, in which the baseline screening method should be a combination of maternal risk factors with MAP.

\section{Strengths and limitations}

Our study compared predictive values of MAP, angiogenic factors and UtA-PI for predicting preeclampsia in a large Asian population. The NORA cohort was a wellperformed prospective study, with standard protocols on measurements of blood pressure, circulating angiogenic factors and UtA-PI. Thus, our results were reliable and comparable with other studies. Prophylactic use of lowdose aspirin was not routinely recommended during study period and three women reported using antihypertensive medication during pregnancy. The incidence of preterm preeclampsia and term preeclampsia was $0.9 \%$ and $1.3 \%$ in the cohort. Thus, our results were less likely affected by the medication issue. However, the exclusion of women with multiple gestations and chronic medical conditions, and lower incidence of preeclampsia in Asian women per se, might have impacts on the relatively low occurrence of preeclampsia in the NORA cohort. On the other hand, our cohort measured blood pressure on one arm and we could not directly compare the performance of MAP from both arms and from one arm. Besides, predictive values of biomarkers at 28-32 weeks might be overestimated, as women who had already been diagnosed as preeclampsia might not be excluded due to unknown time of diagnosis in our study. The potential presence of white coat hypertension might impact on the performance of blood pressure in the prediction of preeclampsia.

\section{Conclusion}

MAP is a good predictor for preeclampsia, especially term preeclampsia, in Asian women. For prediction of preterm preeclampsia, the performance of MAP and PlGF was similar. Our experience indicates that multiple recordings in antenatal blood pressure measurement are encouraged to achieve better accuracy of MAP.

Acknowledgements The authors thank all the NORA cohort (Neonatal and Obstetrics Risks Assessment) participants for their commitment to the study and extend their gratitude to the NORA cohort investigators and staff at KK Women's and Children's Hospital, Singapore for their invaluable assistance for the study.

Contributors JZhu and JZhang conceived the study and provided overall guidance. $\mathrm{BC}$ and KHT collected data. NSR assisted with data collection and conducted the 
statistical analysis. JZhu, JZhang and KHT drafted the manuscript and all authors contributed to interpretation of the results and development of the report.

Funding The NORA study was funded by the Singapore National Medical Research Council (NMRC) Project Grant number NMRC/PPG/KKH/2010-NORA and supported by the NMRC centre grant - Integrated Platform for Research in Advancing Metabolic Health Outcomes of Women and Children (NMRC/CG/ C008A/2017_KKH-IPRAMHO).

Disclaimer The funders did not play any role in the study design, data collection and analysis, decision to publish, or preparation of the manuscript.

Competing interests None declared.

Patient consent for publication Not required.

Ethics approval This study was approved by the SingHealth Centralised Institutional Review Board Ethics Committee, Singapore (CIRB Ref No. 2010/214/D, 4 June 2010)

Provenance and peer review Not commissioned; externally peer reviewed.

Data availability statement All data relevant to the study are included in the article or uploaded as supplementary information.

Supplemental material This content has been supplied by the author(s). It has not been vetted by BMJ Publishing Group Limited (BMJ) and may not have been peer-reviewed. Any opinions or recommendations discussed are solely those of the author(s) and are not endorsed by BMJ. BMJ disclaims all liability and responsibility arising from any reliance placed on the content. Where the content includes any translated material, BMJ does not warrant the accuracy and reliability of the translations (including but not limited to local regulations, clinical guidelines, terminology, drug names and drug dosages), and is not responsible for any error and/or omissions arising from translation and adaptation or otherwise.

Open access This is an open access article distributed in accordance with the Creative Commons Attribution Non Commercial (CC BY-NC 4.0) license, which permits others to distribute, remix, adapt, build upon this work non-commercially, and license their derivative works on different terms, provided the original work is properly cited, appropriate credit is given, any changes made indicated, and the use is non-commercial. See: http://creativecommons.org/licenses/by-nc/4.0/.

ORCID iD

Jun Zhang http://orcid.org/0000-0003-1706-1611

\section{REFERENCES}

1 Duley L. The global impact of pre-eclampsia and eclampsia. Semin Perinatol 2009;33:130-7.

2 Say L, Chou D, Gemmill A, et al. Global causes of maternal death: a WHO systematic analysis. Lancet Glob Health 2014;2:e323-33.

3 LeFevre ML. Low-dose aspirin use for the prevention of morbidity and mortality from preeclampsia: U.S. preventive services task force recommendation statement. Ann Intern Med 2014;161:819-26.

4 The National Institute for Health and Clinical Excellence. Hypertension in pregnancy: diagnosis and management. NICE guideline 133; June 2019.

5 O'Gorman N, Wright D, Poon LC, et al. Multicenter screening for pre-eclampsia by maternal factors and biomarkers at 11-13 weeks' gestation: comparison with NICE guidelines and ACOG recommendations. Ultrasound Obstet Gynecol 2017;49:756-60.

6 O'Gorman N, Wright D, Syngelaki A, et al. Competing risks model in screening for preeclampsia by maternal factors and biomarkers at 11-13 weeks gestation. Am J Obstet Gynecol 2016;214:103.e1-12.

7 O'Gorman N, Wright D, Poon LC, et al. Accuracy of competingrisks model in screening for pre-eclampsia by maternal factors and biomarkers at 11-13 weeks' gestation. Ultrasound Obstet Gynecol 2017;49:751-5.

8 Cheng YKY, Leung TY, Law LW, et al. First trimester screening for pre-eclampsia in Chinese pregnancies: case-control study. BJOG: Int J Obstet Gy 2018;125:442-9.

9 Chaemsaithong P, Pooh RK, Zheng M, et al. Prospective evaluation of screening performance of first-trimester prediction models for preterm preeclampsia in an Asian population. Am J Obstet Gynecol 2019;221:650.e1-650.e16.

10 Poon LC, Shennan A, Hyett JA, et al. The International Federation of gynecology and obstetrics (FIGO) initiative on pre-eclampsia: a pragmatic guide for first-trimester screening and prevention. Int $J$ Gynaecol Obstet 2019;145:1-33.

11 Cnossen JS, Vollebregt KC, de Vrieze N, et al. Accuracy of mean arterial pressure and blood pressure measurements in predicting pre-eclampsia: systematic review and meta-analysis. BMJ 2008;336:1117-20.

12 Gasse C, Boutin A, Coté M, et al. First-trimester mean arterial blood pressure and the risk of preeclampsia: the great obstetrical syndromes (GOS) study. Pregnancy Hypertens 2018;12:178-82.

13 Chaemsaithong P, Sahota D, Pooh RK, et al. First-trimester preeclampsia biomarker profiles in Asian population: multicenter cohort study. Ultrasound Obstet Gynecol 2020;56:206-14.

14 Wataganara T, Leetheeragul J, Pongprasobchai S, et al. Prediction and prevention of pre-eclampsia in Asian subpopulation. J Obstet Gynaecol Res 2018;44:813-30.

$15 \mathrm{Ng}$ QJ, Zhang J, Dai F, et al. Neonatal and obstetric risk assessment (NorA) pregnancy cohort study in Singapore. Int J Gynaecol Obstet 2018;4:31-7.

16 Brown MA, Lindheimer MD, de Swiet M, et al. The classification and diagnosis of the hypertensive disorders of pregnancy: statement from the International Society for the study of hypertension in pregnancy (ISSHP). Hypertens Pregnancy 2001;20:ix-xiv.

17 Plasencia W, Maiz N, Poon L, et al. Uterine artery Doppler at $11+0$ to $13+6$ weeks and $21+0$ to $24+6$ weeks in the prediction of preeclampsia. Ultrasound Obstet Gynecol 2008;32:138-46.

18 Khalil A, Garcia-Mandujano R, Maiz N, et al. Longitudinal changes in uterine artery Doppler and blood pressure and risk of pre-eclampsia. Ultrasound Obstet Gynecol 2014;43:541-7.

19 O'Gorman N, Tampakoudis G, Wright A, et al. Uterine artery pulsatility index at 12, 22, 32 and 36 weeks' gestation in screening for pre-eclampsia. Ultrasound Obstet Gynecol 2016;47:565-72.

20 Plasencia W, Maiz N, Bonino S, et al. Uterine artery doppler at 11 +0 to $13+6$ weeks in the prediction of pre-eclampsia. Ultrasound Obstet Gynecol 2007;30:742-9.

21 Albaiges $\mathrm{G}$, Missfelder-Lobos $\mathrm{H}$, Lees $\mathrm{C}$. One-stage screening for pregnancy complications by color Doppler assessment of the uterine arteries at 23 weeks' gestation. Obstet Gynecol 2000;96:559-64.

22 Pickering TG, Hall JE, Appel LJ, et al. Recommendations for blood pressure measurement in humans and experimental animals: part 1: blood pressure measurement in humans: a statement for professionals from the subcommittee of professional and public education of the American heart association council on high blood pressure research. Circulation 2005;111:697-716.

23 DeLong ER, DeLong DM, Clarke-Pearson DL. Comparing the areas under two or more correlated receiver operating characteristic curves: a nonparametric approach. Biometrics 1988;44:837-45.

24 Poon LCY, Kametas N, Strobl I, et al. Inter-arm blood pressure differences in pregnant women. BJOG 2008;115:1122-30.

25 Poon LC, Kametas NA, Pandeva I, et al. Mean arterial pressure at $11(+0)$ to $13(+6)$ weeks in the prediction of preeclampsia. Hypertension 2008;51:1027-33.

26 Poon LCY, Zymeri NA, Zamprakou A, et al. Protocol for measurement of mean arterial pressure at 11-13 weeks' gestation. Fetal Diagn Ther 2012;31:42-8.

27 McElrath TF, Lim K-H, Pare E, et al. Longitudinal evaluation of predictive value for preeclampsia of circulating angiogenic factors through pregnancy. Am J Obstet Gynecol 2012;207:407.e1-e7.

28 Kusanovic JP, Romero R, Chaiworapongsa T, et al. A prospective cohort study of the value of maternal plasma concentrations of angiogenic and anti-angiogenic factors in early pregnancy and midtrimester in the identification of patients destined to develop preeclampsia. J Matern Fetal Neonatal Med 2009:22:1021-38.

29 Taylor K, Ferreira DLS, West J,et al. Differences in pregnancy metabolic profiles and their determinants between white European and South Asian women: findings from the born in Bradford cohort. Metabolites 2019;9:190.

30 Abbasi H, Dastgheib SA, Hadadan A, et al. Association of Endothelia Nitric Oxide Synthase 894G > T Polymorphism with Preeclampsia Risk: A Systematic Review and Meta-Analysis based on 35 Studies. Fetal Pediatr Pathol 2020;15:1-16.

31 Veisian M, Tabatabaei RS, Javaheri A, et al. Association of Interleukin-10 -1082G > A Polymorphism with Susceptibility to Preeclampsia: A Systematic Review and Meta-Analysis Based on 21 Studies. Fetal Pediatr Pathol2019:1-15.

32 Steegers EAP, von Dadelszen P, Duvekot JJ, et al. Pre-eclampsia. Lancet 2010;376:631-44. 